Jyh-Ping Hsu'
Shih-Wei Huang ${ }^{1}$
Tien-Shiang Hsieh ${ }^{1}$
Tai-Horng Young
Wei-Wen Hu'

'Department of Chemical
Engineering
'Institute of Biomedical
Engineering, College of Medicine
and College of Engineering,
National Taiwan University,
Taipei, Taiwan, ROC

Jyh-Ping Hsu

Tai-Horng Young ${ }^{2}$

Department of Chemical

Institute of Biomedical

Engineering, College of Medicine

Taipei, Taiwan, ROC

\section{Electrophoretic behavior of cerebellar granule neurons}

The electrophoresis of cerebellar granule neurons is observed, and a theoretical model proposed to simulate its electrophoretic behavior. We assume that the surface of a neuronal cell carries dissociable acidic functional groups, and the liquid phase contains a mixed $(a: b)+(c: b)$ electrolyte, where $a$ and $c$ are the valences of cations and $b$ is the valence of anions. The cations of valence $c$ are allowed to bind to dissociated functional groups. The model proposed is readily applicable to the prediction of the surface properties of cerebellar granule neurons such as the density of dissociable functional groups and the equilibrium constant of the dissociation reaction. The applicability of the present model is justified by fitting it to the measured electrophoretic mobility data.

Keywords: Cerebellar granule neurons / Electrophoretic mobility / lon-binding / Surface dissociation reaction / Surface properties

\section{Introduction}

Electrophoresis, the movement of a charged entity in a response to an applied electric field, is an efficient and convenient tool for both description and quantification of its surface properties, in particular, its charged conditions such as the sign of surface charge and the level of surface potential. The theoretical basis for electrophoresis was founded by Smoluchowski [1] in which the relation between the electrophoretic velocity of an entity and the applied electric field was derived. The original derivation of Smoluchowski was based on a rigid particle with a constant surface potential in a uniform electric field. For the case of biocolloids, their surface charge often arises from the dissociation of the functional groups they carry [2-6]. In this case, rather than remains at constant electrical potential, the surface condition becomes more complicated, and the classic result of Smoluchowski needs to be modified accordingly. To this end, various models have been proposed in the literature. Levine et al. [5], for example, investigated theoretically the electrophoresis of human erythrocytes. They concluded that the mobility of erythrocytes is significantly smaller than that predicted by the Smoluchowski's formula. Ohshima and Kondo [7] analyzed the electrophoretic behavior of a particle coated with an ion-penetrable membrane layer having uniformly distributed fixed charge. Their model was extended by Hsu et al. [8] to the case of linear and exponential distributed fixed charge. Ohshima[9] derived a general expression for the electrophoretic mobility of a rigid sphere covered by

Correspondence: Prof. Dr. Jyh-Ping Hsu, Department of Chemical Engineering, National Taiwan University, Taipei, Taiwan 10617, ROC

E-mail: jphsu@ccms.ntu.edu.tw

Fax: $+886-2-23623040$ a polyelectrolyte layer. The analyses of Ohshima and Kondo [7] and Hsu et al. [8] were extended by Hsu and Fan [10] to take the effect of the spatial variations in both the fixed charge and the dielectric constant in the membrane layer into account. Hsu et al. [11] estimated the electrophoretic mobility of a planar particle covered by an ionpenetrable membrane in a solution containing asymmetric electrolyte by assuming a uniform fixed charge distribution. A more detailed analysis was conducted by Tseng et al. [12] to take the effect of the degree of dissociation of the functional groups in the membrane layer into account.

Employing a microelectrophoresis technique, Mironov and Dolgaya [13] studied experimentally the surface charge of isolated rat dorsal root ganglion neurons. Their results showed that the mobility increases with the increase in $\mathrm{pH}$, and decreases with the increase in electrolyte concentration. They suggested that the outer surface of a neuron cell contains dissociable functional groups, and the $\mathrm{Ca}^{2+}$ in the liquid phase may bind to dissociated functional groups. Kawahata et al. [14] examined the charge distribution in the surface region for human erythrocytes of groups $\mathrm{A}, \mathrm{B}, \mathrm{AB}$, and $\mathrm{O}$ from their electrophoretic mobility data at various $\mathrm{pH}$ and ionic strength of the medium. The electrophoretic behaviors of rat lymphocyte subpopulations were examined by Morita et al. [15]; the electrophoretic mobility of both rat $B$ cells and T cells at various $\mathrm{pH}$ and ionic strength were measured, and the average dissociation constants of the acidic and basic groups in the surface region were estimated. Makino et al. [16] studied the surface properties of rat basophilic leukemia cells (RBL) through electrophoretic measurements. Their results showed that RBL change their functional properties when they adhere to a solid substrate and the negative charge density in the surface layer increases. Hayashi et al. [17] examined the surface properties of 
bacterial cells by electrophoretic mobility measurement. They showed that the surface potential of a cell estimated based on a soft-particle model is lower than that based on the Smoluchowski's formula.

During the past several decades, a tremendous effort has been directed toward the development of methods for replacing lost or dysfunctional neurons following trauma or disease by means of tissue transplantation or peripheral nerve grafting [18-22]. A detailed understanding of the behavior of neuronal cell has attracted much attention because of its clinical importance and scientific interest. A critical element in the design of strategies for studying the behavior of neuronal cell is to understand the factors that influence cellular mechanism. Since the first point of contact between a cell and other cells or substrates is its membrane layer, cell behavior is highly dependent upon the properties of this layer. One of the parameters reflecting the state of cell membrane is its surface charge, which influences the concentration of biologically active substances of medium adjacent to the cell surface and in turn, influences cell-to-cell and cell-to-substrate interactions. In addition, the surface charge depends also on the intracellular metabolism [13], thus, changes in the charge of outer membrane surface may be used to investigate the synthesis and reconstruction of the intracellular components. Although there are several papers published for the measurement of the surface charge of different cells by employing a microelectrophoresis technique [14-17], their principle research efforts are dedicated to non-neuronal cells. Up to now, only few studies have been devoted to the cell surface properties in relation to neuron cells in the electrophoresis process [13].

In the present study, the electrophoresis of cerebellar granule neurons is observed through an electrophoresis chamber, and a theoretical model is proposed to simulate its electrophoretic behavior. The granule neurons are chosen since they can be prepared easily from postnatal rat cerebellum [23]. On the basis of the model proposed by Hsu and Kuo [24], we consider a general case where the surface of a neuronal cell carries dissociable function groups and the liquid phase contains a mixed electrolyte. Also, the multivalent counterions in the liquid phase are allowed to bind to the dissociated functional groups on the surface of a neuronal cell.

\section{Materials and methods}

\subsection{Experimental conditions}

Cerebellar granule neurons from 7-day-old Wistar rats were used in this study. Briefly, by mechanical disruption in the presence of trypsin and DNAse [25], neurons were

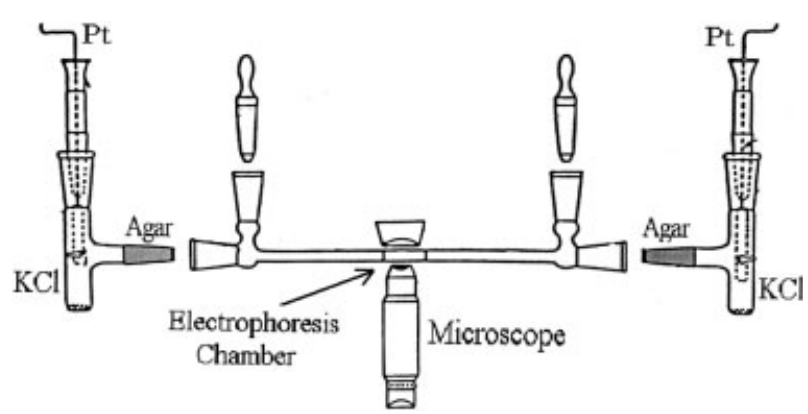

Figure 1. Schematic representation of the apparatus [2] used to observe the electrophoresis of cerebellar granule neurons.

dissociated from freshly dissected cerebella. Subsequently, neurons were suspended in basal Eagle's medium (BME; Gibco BRL, Grand Island, NY, USA) supplemented with $10 \%$ fetal bovine serum (FBS; Gibco), $25 \mathrm{~mm} \mathrm{KCl}$, penicillin $\mathrm{G}(100 \mathrm{IU} / \mathrm{mL})$ and streptomycin $(100 \mu \mathrm{g} / \mathrm{mL})$. Prior to the neuronal mobility measurement, the ionic strength and $\mathrm{pH}$ of cell suspension was adjusted by dilution of the cell suspension with a solution containing $\mathrm{KCl}, \mathrm{CaCl}_{2}$ and Tris- $\mathrm{HCl}$. All solutions used were made isotonic by addition of glucose. The electrophoretic behavior of neurons was measured by introducing neurons at average density of $5 \times 10^{3}$ cells $/ \mathrm{mL}$ into a rectangular glass electrophoresis chamber similar to that used by Mironov and Dolgaya [13], a schematic representation of which is illustrated in Fig. 1. The length, width, and height of the chamber are $5 \mathrm{~cm}, 1 \mathrm{~cm}$, and $0.1 \mathrm{~cm}$, respectively. Agar bridges (1\% agar) were placed in the ends of the chamber and connected the DC supply (200 V) with two compartments containing Pt electrodes in saturated $\mathrm{KCl}$ solution. The hole for introducing cell suspension was located in the upper side of the chamber and was close to the agar bridge. Current passing through the chamber ranged from 3 to $10 \mathrm{~mA}$.

The electrophoretic behavior of cerebellar granule neurons was observed by using an inverted microscope attached with a CCD camera. The objective of the microscope was focused on neurons located in the central but slightly lower stationary level of the chamber, selected so as to give a stable electrophoretic behavior of neurons. All measurements were conducted at $22 \pm 2^{\circ} \mathrm{C}$ and their duration did not exceed $15 \mathrm{~min}$ to avoid the temperature increase of the chamber due to the heating effect of the microscope illumination. The magnitude of the velocity of cerebellar granule neurons $u$ was measured by recording the time needed for them to pass a fixed number of gratitude divisions (ca. $100 \mu \mathrm{m}$ ). The electrophoretic mobility $\mu$ was calculated by $\mu=u / E=u g S / l$, where $E$ is the strength of the applied electric field, $S$ is the cross-sectional area of the observation chamber, $l$ is the current, and $g$ is the 
Table 1. Experimental conditions at which the electrophoretic behavior of cerebellar granule neurons is observed.

\begin{tabular}{lll}
\hline $\mathrm{pH}$ & $g(\mathrm{~mA} / \mathrm{mV} \mathrm{cm})$ & $I(\mathrm{~mA})$ \\
\hline 4.5 & 1.390 & 3.140 \\
5 & 1.346 & 2.980 \\
6 & 1.485 & 2.920 \\
7 & 1.747 & 3.475 \\
8 & 1.841 & 3.505 \\
9 & 1.901 & 3.600 \\
\hline
\end{tabular}

The results obtained are summarized in Fig. 3. $S=0.13 \mathrm{~cm}^{2}$

Table 2. Experimental conditions at which the electrophoretic behavior of cerebellar granule neurons is observed.

\begin{tabular}{ccll}
\hline pH 9 & $\mathrm{CaCl}_{2}(\mathrm{~mm})$ & $g(\mathrm{~mA} / \mathrm{mV} \mathrm{cm})$ & $I(\mathrm{~mA})$ \\
\cline { 2 - 4 } & 0.2 & 1.702 & 3.355 \\
& 0.5 & 1.540 & 3.035 \\
& 2.0 & 1.807 & 3.475 \\
& 5.0 & 2.530 & 4.245 \\
& 10.0 & 3.110 & 4.360 \\
\hline $\mathrm{pH} 5$ & 0.2 & 1.074 & 3.010 \\
& 0.5 & 1.150 & 3.340 \\
& 2.0 & 1.463 & 4.110 \\
& 5.0 & 1.907 & 5.290 \\
& 10.0 & 2.710 & 8.600 \\
\hline
\end{tabular}

The results obtained are summarized in Fig. 4.

$\mathrm{S}=0.13 \mathrm{~cm}^{2}$

conductivity of solution. Each reported mobility was based on ten readings (i.e., neuron cells); the standard error is below $5 \%$. A basic solution containing $2.5 \mathrm{~mm} \mathrm{KCl}$, $2.0 \mathrm{mM} \mathrm{CaCl}_{2}, 10 \mathrm{~mm}$ Tris- $\mathrm{HCl}$, and $280 \mathrm{~mm}$ glucose is used in most of our experiments. The $\mathrm{pH}$ is adjusted by the addition of either $\mathrm{NaOH}_{(\mathrm{aq})}$ or $\mathrm{HCl}_{(\mathrm{aq})}$. Experimental details are summarized in Tables 1 and 2.

\subsection{Theory}

Suppose that the linear size of a neuronal cell is much larger than the thickness of the double layer surrounding it, and the local curvature of cell surface is negligible. In this case, the neuronal cell can be assumed to be planar, that is, only a one-dimensional problem needs to be considered. The liquid phase contains a mixed $(a: b)+(c: b)$ electrolyte where $a$ and $c$ are the valences of cations and $b$ is the valence of anions. Referring to Fig. 2, an electric field $E$ parallel to cell surface is applied, and the cell moves with velocity $U_{0}$ at steady state. The problem under consideration involves solving simultaneously the equations describing the electrical field and that describing the flow field.

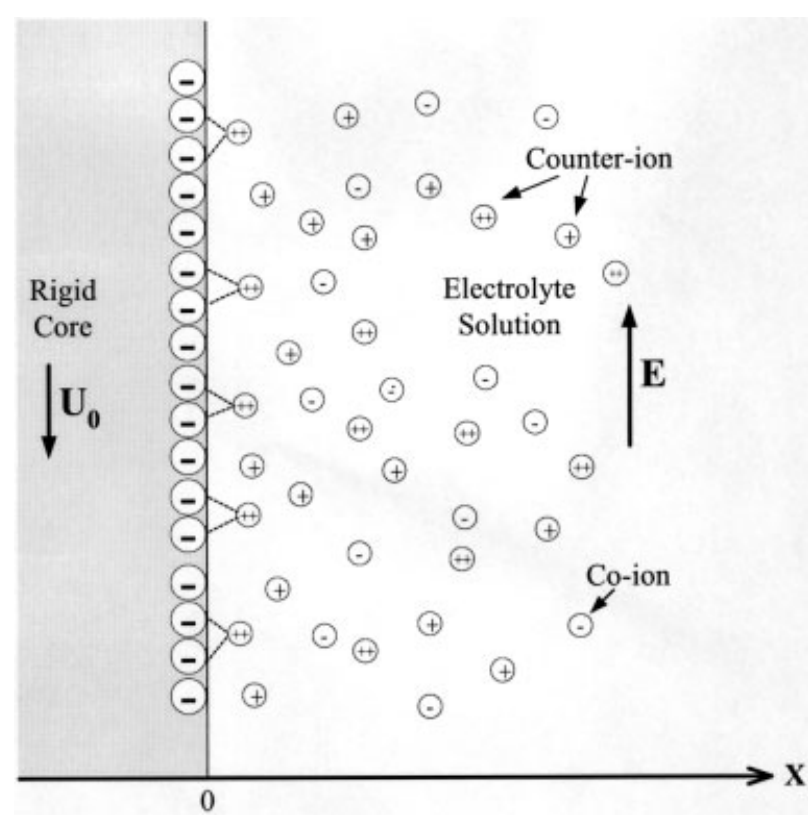

Figure 2. Schematic representation of the problem considered. An electric field $E$ parallel to the surface of a neuron cell is applied, $U_{0}$ is the electrophoretic velocity of neuron cell, and $X$ is the scaled distance from cell surface.

\subsubsection{Electrical field}

Suppose that the spatial variation in the electrical potential can be described by the Poisson-Boltzmann equation [24]

$\frac{\mathrm{d}^{2} \phi}{\mathrm{d} X^{2}}=\frac{\left[e^{\mathrm{b} \phi}-(1-\xi) e^{-\mathrm{a} \phi}-\zeta e^{-c \phi}\right]}{(a+b)+(c-a) \xi}$

where

$\phi=e \phi / k_{\mathrm{B}} T$

$\xi=c n_{\mathrm{c}}^{0} / b n_{\mathrm{b}}^{0}$

$\kappa^{2}=e^{2}\left[a(a+b) n_{\mathrm{a}}^{0}+c(b+c) n_{\mathrm{c}}^{0}\right] / \varepsilon_{0} \varepsilon_{\mathrm{r}} k_{\mathrm{B}} T$

$X=\kappa r$

$b n_{\mathrm{b}}^{0}=a n_{\mathrm{a}}^{0}+c n_{\mathrm{c}}^{0}$

In these expressions, $\phi$ is the electrical potential, $\xi$ is the fraction of cations of valence $c$ in the bulk liquid phase, $\mathrm{n}_{\mathrm{a}}{ }^{0}, \mathrm{n}_{\mathrm{b}}{ }^{0}$, and $\mathrm{n}_{\mathrm{c}}{ }^{0}$ are, respectively, the number concentrations of ionic species of valences $a,-b$, and $c$ in the bulk liquid phase, $e$ is the elementary charge, $\varepsilon_{r}$ and $\varepsilon_{0}$ are respectively the relative permittivity of the liquid phase and the permittivity of a vacuum, $k_{\mathrm{B}}$ and $T$ denote, respectively, the Boltzmann constant and the absolute temperature, and $\kappa$ and $r$ being, respectively, the reciprocal Debye length and the distance from cell surface. $\varphi$ and $X$ are 
respectively the scaled electrical potential and the scaled distance from cell surface.

We assume that the surface of a neuronal cell contains acidic functional group, HA, which is capable of undergoing the dissociation reaction

$\mathrm{HA} \leftrightarrow \mathrm{H}^{+}+\mathrm{A}^{-}$

Let $K_{\mathrm{a}}$ be the dissociation constant of this reaction. Then

$K_{\mathrm{a}}=\frac{C_{\mathrm{H}^{+}}^{\mathrm{s}}+C_{\mathrm{A}^{-}}}{C_{\mathrm{HA}}}$

where $C_{\mathrm{HA}}$ and $C_{\mathrm{A}^{-}}$are respectively the surface concentration of $\mathrm{HA}$ and that of $\mathrm{A}^{-}$, and $\mathrm{C}_{\mathrm{H}^{+}}^{\mathrm{s}}$ is the concentration of $\mathrm{H}^{+}$on particle surface. Suppose that the spatial distribution of $\mathrm{H}^{+}$follows the Boltzmann distribution. Then

$C_{\mathrm{H}^{+}}^{\mathrm{S}}=C_{\mathrm{H}^{+}}^{0} \exp (-\phi)$

where $C_{\mathrm{H}^{+}}^{0}$ is the bulk concentration of $\mathrm{H}^{+}$.

Without loss of generality, we assume that $c$ takes the value of two, that is, the liquid phase contains a divalent cation, $\mathrm{M}^{2+}$. Suppose that this cation is capable of binding to $A^{-}$through the following expression:

$2 \mathrm{~A}^{-}+\mathrm{M}^{2+} \leftrightarrow \mathrm{MA}_{2}$

The equilibrium constant of this expression, $K_{\mathrm{b}}$, can be expressed as

$K_{\mathrm{b}}=\frac{C_{\mathrm{MA}_{2}}}{\left(C_{\mathrm{A}^{-}}\right)^{2} C_{\mathrm{M}^{2+}}^{\mathrm{S}}}$

where $C_{\mathrm{MA}_{2}}$ and $C_{\mathrm{M}^{2+}}^{\mathrm{S}}$ are respectively the surface concentration of $\mathrm{MA}_{2}$ and that of $\mathrm{M}^{2+}$ on particle surface. If we assume that the spatial variation in the concentration of $\mathrm{M}^{2+}$ follows the Boltzmann distribution, then

$C_{\mathrm{M}^{2+}}^{\mathrm{S}}=C_{\mathrm{M}^{2+}}^{0} \exp (-2 \phi)$

$C_{\mathrm{M}^{2+}}^{0}$ being the bulk concentration of $\mathrm{M}^{2+}$. If we let $N_{\mathrm{s}}$ be the density of the acidic dissociation functional groups on particle surface, then

$N_{\mathrm{s}}=C_{\mathrm{A}^{-}}+C_{\mathrm{HA}}+C_{\mathrm{MA}_{2}}$

It can be shown that Eqs. (2) - (8) lead to (see Appendix)

$C_{\mathrm{A}^{-}}=\frac{\sqrt{y^{2}+4 K_{\mathrm{b}} C_{\mathrm{M}^{2+}} N_{\mathrm{s}}}-y}{2 K_{\mathrm{b}} C_{\mathrm{M}^{2+}}}$

where

$y=1+C_{\mathrm{H}^{+}} / K_{\mathrm{a}}$

The fixed charge density on particle surface, $\sigma$, can be expressed as

$\sigma=F C_{\mathrm{A}^{-}}$

where $F$ is the Faraday constant.
If we assume that the electrical potential vanishes at a point far away from cell surface, then boundary conditions associated with Eq. (1) can be expressed as

$\frac{\mathrm{d} \phi}{\mathrm{d} X}=\Gamma$ as $X \rightarrow 0$

$\frac{\mathrm{d} \phi}{\mathrm{d} X} \rightarrow 0$ as $X \rightarrow \infty$

where $\Gamma=-e \sigma / \varepsilon_{0} \varepsilon_{\mathrm{r}} k_{\mathrm{B}} T \kappa$ is the scaled surface charge density.

\subsubsection{Flow field}

Suppose that the flow field can be described by the scaled Navier-Stokes equation

$\frac{\mathrm{d}^{2} U}{\mathrm{~d} X^{2}}=L \frac{e^{\mathrm{b} \phi}-(1-\xi) e^{-\mathrm{a} \phi}-\xi e^{-c \phi}}{(a+b)+(c-a) \xi}$

where

$U=u / U_{0}$

$L=\varepsilon_{0} \varepsilon_{\mathrm{r}} k_{\mathrm{B}} T E / \eta \mathrm{e} U_{0}$

In these expressions, $u$ and $U_{0}$ are respectively the magnitude of the velocity of the liquid phase, $U_{0 .}, U$ and $E$ are respectively the magnitude of the scaled velocity and the strength of the applied electric field, and $\eta$ is the viscosity of the liquid phase. For convenience, a neuron cell is held fixed and the liquid moves in the inverse direction as that of $U_{0}$. Therefore, the boundary conditions associated with Eq. (13) are assumed as

$U=0$ as $X \rightarrow 0$

$U \rightarrow-1$ as $X \rightarrow \infty$

Equation (15) implies that the slipping plane is chosen at the cell surface. Solving Eqs. (1) and (13) simultaneously subject to the boundary conditions expressed in Eqs. (11) - (12), (14), and (15) provides the spatial variation of electrical potential and that of fluid velocity. The electrophoretic mobility, $\mu$, defined by $\mu=U_{0} / E$, can then be evaluated based on these results.

\section{Results and discussion}

Figure 3 shows the variation of the mobility of cerebellar granule neurons $\mu$ as a function of the $\mathrm{pH}$ of the bulk liquid phase. The discrete symbols represent experimental data, and the curves denote the results predicted by our model, where the adjustable parameters are estimated through regression analysis. The sign of the mobility reveals that the surface of cerebellar granule neurons is 


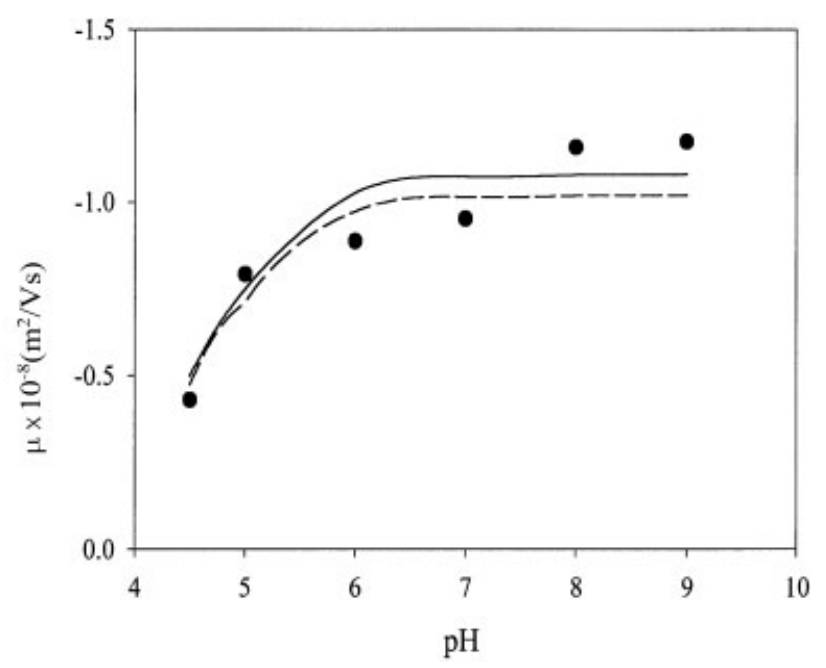

Figure 3. Variation of mobility $\mu$ as a function of $\mathrm{pH}$. Discrete symbols represent experimental data, and curves denote simulated results based on present model. Solid curve, $N_{\mathrm{s}}=3.3 \times 10^{-8} \mathrm{~mol} / \mathrm{m}^{2}$, dashed curve, $N_{\mathrm{s}}=3.1 \times$ $10^{-8} \mathrm{~mol} / \mathrm{m}^{2}$. Key: $T=298 \mathrm{~K}, \varepsilon_{\mathrm{r}}=78.5, \eta=8.91 \times 10^{-4}$ $\mathrm{Ns} / \mathrm{m}^{2}, a=b=1, c=2, \xi=0.6, E=1200 \mathrm{~V} / \mathrm{m}, K_{\mathrm{a}}=3 \times$ $10^{-5} \mathrm{M}$, and $K_{\mathrm{b}}=10.0 \mathrm{~m}^{5} / \mathrm{mol}^{2}$.

negatively charged, which is typical to biological cells. As can be seen in Fig. 3, $|\mu|$ increases with the increase in $\mathrm{pH}$ for $\mathrm{pH}<7$, and becomes roughly constant for $\mathrm{pH}>8$, which is consistent with the experimental observations in the literature $[13,17]$. This is because if $\mathrm{pH}$ is low, the degree of dissociation of the acidic functional groups on the surface of cerebellar granule neurons is low, which leads to a low concentration of negative fixed charge, and therefore, a small mobility. On the other hand, if $\mathrm{pH}$ is sufficiently high, the dissociation of the acidic functional groups is almost complete, and the concentration of negative fixed charge remains essentially constant, so is the mobility.

Figure 4 shows the variation of the mobility of cerebellar granule neurons $\mu$ as a function of the concentration of $\mathrm{M}^{2+}$, which is $\mathrm{Ca}^{2+}$ in the present case, at two levels of $\mathrm{pH}$. The experimental data are represented by discrete symbols, and the theoretical calculations are illustrated as curves with the adjustable parameters estimated through regression analysis. Here, the total number of acidic functional groups on the surface of cerebellar granule neurons is fixed. Figure 4 reveals that $|\mu|$ decreases with the increase in the concentration of $\mathrm{Ca}^{2+}$ in the liquid phase. This is because raising the concentration of $\mathrm{Ca}^{2+}$ has the effect of increasing its probability to bind to the dissociated functional groups on cell surface, thereby reducing the surface charge density. As in the case of Fig. 3, the mobility increases with the increase in $\mathrm{pH}$.

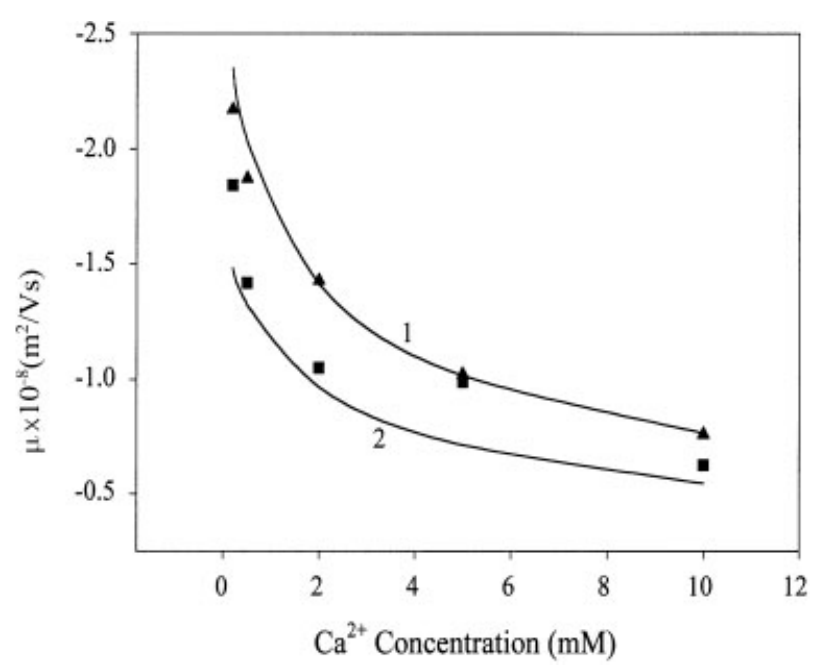

Figure 4. Variation of mobility $\mu$ as a function of the concentration of $\mathrm{Ca}^{2+}$ at two levels of $\mathrm{pH}$. Discrete symbols represent experimental data: $(\boldsymbol{\Delta}) \mathrm{pH}$ 9; $(\boldsymbol{\square}), \mathrm{pH} 5$. Solid curves, results based on present model for the case $N_{\mathrm{s}}=$ $4.5 \times 10^{-8} \mathrm{~mol} / \mathrm{m}^{2}$. Curve 1, $\mathrm{pH} \mathrm{9,2,} \mathrm{pH}$ 5. Key: same as in Fig. 3.

\section{Concluding remarks}

The electrophoretic behavior of cerebellar granule neurons is investigated. In addition to a direct experimental observation, a theoretical model, which is based on an ion-binding mechanism on cell surface, is proposed to elaborate the general trend of the experimental data gathered. It has the advantage that the surface properties of cerebellar granule neurons, such as the charge density and the rate constant for the dissociation of functional groups, can be estimated through electrophoretic measurements. The model proposed is also applicable to the prediction of the effects of $\mathrm{pH}$ and the presence of multivalent counterions in the liquid phase on the electrophoretic behavior. These are highly desirable to cell biologists for practical considerations. We show that the absolute electrophoretic mobility of a neuron cell increases with the increase in $\mathrm{pH}$ for $\mathrm{pH}<7$, and becomes essentially constant for $\mathrm{pH}>8$. Increasing the concentration of multivalent counterions in the liquid phase has the effect of increasing its probability to bind to the dissociated functional groups on cell surface, thereby reducing its electrophoretic mobility.

This work is supported by the National Science Council of the Republic of China.

Received November 12, 2001 


\section{References}

[1] Hunter, R. J., Foundations of Colloid Science, Oxford University Press, Oxford 1989, Vol. 1.

[2] Seaman, G. V. F., Heard, D. H., Blood 1961, 18, 599-604.

[3] Haydon, D. A., Seaman, G. V. F., Arch. Biochem. Biophys. 1967, 122, 126-136.

[4] Seaman, G. V. F., Vassar, P. S., Kendal, M.J., Arch. Biochem. Biophys. 1969, 135, 356-362.

[5] Levine, S., Levine, M., Sharp, K. A., Brooks, D. E., Biophys. J. 1983, 42, 127-135.

[6] Sharp, K. A., Brooks, D. E., Biophys. J. 1985, 47, 563-566.

[7] Ohshima, H., Kondo, T., J. Colloid Interface Sci. 1987, 116, 305-311.

[8] Hsu, J. P., Hsu, W. C., Chang, Y. I., Colloid Polym. Sci. 1994, 272, 251-260.

[9] Ohshima, H., J. Colloid Interface Sci. 1994, 163, 474-483.

[10] Hsu, J. P., Fan, Y. P., J. Colloid Interface Sci. 1995, 172, 230241.

[11] Hsu, J. P., Lin, S. H., Tseng, S., J. Theor. Biol. 1996, 182, 137-145.

[12] Tseng, S., Lin, S. H., Hsu, J. P., Colloids Surfaces B 1999, 13, 277-286.

[13] Mironov, S. L., Dolgaya, E. V., J. Membr. Biol. 1985, 86, 197202.

[14] Kawahata, S., Ohshima, H., Muramatsu, N., Kondo, T., J. Colloid Interface Sci. 1990, 138, 182-186.

[15] Morita, K., Muramatsu, N., Ohshima, H., Kondo, T., J. Colloid Interface Sci. 1991, 147, 457-461.

[16] Makino, K., Fukai, F., Kawaguchi, T., Ohshima, H., Colloids Surfaces B 1995, 5, 221-226.

[17] Hayashi, H., Tsuneda, S., Hirata, A., Sasaki, H., Colloids Surfaces $B$ 2001, 22, 149-157.

[18] Yavin, E., Yavin, Z., J. Cell Biol. 1974, 62, 540-546.
[19] Carbonetto, S. T., Gruver, M. M., Turner, D. C., Science 1982, 216, 897-899.

[20] Aebischer, P., Valentini, R. F., Dario, P., Domenici, C., Galletti, P. M., Brain Res. 1987, 436, 165-168.

[21] Langone, F., Lora, S., Veronese, F. M., Caliceti, P., Valenti, F., Palma, G., Biomaterials 1995, 16, 347-353.

[22] Shoichet, M. S., Winn, S. R., Athavale, S. J., Harris, M., Gentile, F. T., Biotech. Bioeng. 1994, 43, 563-572.

[23] Burgoyne, R. D., Cambray-Deakin, M. A., Brain Res. Rev. 1988, 13, 77-101.

[24] Hsu, J. P., Kuo, Y. C., J. Colloid Interface Sci. 1996, 183, 194-198.

[25] Levi, G., Aloisi, F., Ciotti, M. T., Gallo, V., Brain Res. 1984, 290, 77-86.

\section{Appendix}

Equations (3) and (6) can be rewritten as

$C_{\mathrm{HA}}=\frac{C_{\mathrm{H}^{+}}^{\mathrm{s}}}{K_{\mathrm{a}}} C_{\mathrm{A}^{-}}$

$C_{\mathrm{MA}_{2}}=K_{\mathrm{b}} C_{\mathrm{M}^{2+}}^{\mathrm{s}}\left(C_{\mathrm{A}^{-}}\right)^{2}$

Substituting these expressions into Eq. (8) in the text, we obtain

$N_{\mathrm{s}}=C_{\mathrm{A}^{-}}+\frac{C_{\mathrm{H}^{+}}^{\mathrm{s}}}{K_{\mathrm{a}}} C_{\mathrm{A}^{-}}+K_{\mathrm{b}} C_{\mathrm{M}^{2+}}^{\mathrm{s}}\left(C_{\mathrm{A}^{-}}\right)^{2}$

Equation (A3) can be rewritten as

$K_{\mathrm{b}} C_{\mathrm{M}^{2+}}^{\mathrm{s}}\left(C_{\mathrm{A}^{-}}\right)^{2}+\left(1+\frac{C_{\mathrm{H}^{+}}^{\mathrm{s}}}{K_{\mathrm{a}}}\right) C_{\mathrm{A}^{-}}-N_{\mathrm{s}}=0$

Solving Eq. (A4) for $C_{A^{-}}$, Eq. (9) in the text can be recovered. 